\title{
CATHETER ABLATION OF VENTRICULAR TACHYCARDIA - LONG-TERM RESULTS
}

\author{
Martin Fiala, Petr Heinc, Jan Lukl
}

\author{
I. Department of Medicine, Palacký University, I. P. Pavlova 6, 77520 Olomouc, Czech Republic, Martin.Fiala@fnol.cz
}

Received August 27, 2000

Key words: Ventricular tachycardia / Radiofrequency catheter ablation

Ventricular tachycardia represents life-threatening cardiac rhythm disturbance and catheter ablation using radiofrequency current provides powerful means for definitive cure of almost all monomorphic tachyarrhythmias. Presence and extent of underlying structural heart disease is important for further prognosis of the patients, who undergo catheter ablation of ventricular tachycardia. Long-term results of catheter ablation for ventricular tachycarida in patients with and without structural heart disease are presented.

Patients and results: Twenty three patients (9 females) aged $49.1 \pm 15.6$ (18-72) years underwent catheter ablation for monomorphic (resp. polymorphic in one patient) ventricular tqachycardia in 30 ablation procedures. Patients with structural heart disease: Seven patients (2 females) aged $54.2 \pm 19.8$ (21-72) years had structural heart disease ( 5 patients - post myocardial infarction, 1 patient - arrhythmogenic right ventricular dysplasia, 1 patient - surgically corrected transposition of great arteries). All sustained monomorphic ventricular tachycardias were eliminated during the catheter ablation and no ventricular tachycardia recurred during the follow-up period in 4 patients. In two patients sustained monomorphic ventricular tachycardia was not eliminated with radiofrequency current. One of the patients remains free of ventricular tachycardia and one patient experienced one recurrence of slowed ventricular tachycardia. Thus long-term clinical success was achieved in 4 patients and some clinical benefit probably also in the latter two patients. A different ablation strategy targeting large arrhythmogenic area at the border of postmyocardial infarction scar was employed in the last patient with frequent ICD discharges for polymorphic ventricular tachycardia associated with hemodynamic deterioration. This procedure brought immediate and long-term significant reduction of ICD shocks and rehospitalizations and probably was life-saving. Patients without structural heart disease: In sixteen patients (7 females) aged $44.2 \pm 12.8$ (18-66) years no structural heart disease was found. These patients presented with documented ventricular ectopy in different forms from incessant ventricular premature beats through repetitive nonsustained ventricular tachycardia to paroxysmal sustained ventricular tachycardia. The arrhythmia originated in the right ventricle in 11 patients (right ventricular outflow tract in 10 patients and basolateral wall in 1 patient) and in the left ventricle in 5 patients (inferoapicoseptal region in 4 patients and basoinferoseptal region in 1 patient). Eleven patients $(68.7 \%)$ had the arrhythmia eliminated or markedly suppressed during the ablation procedure and remain free of palpitations and antiarrhythmic drugs. Two patients with partial suppression of the ectopic rhythm are less symptomatic and the antiarrhythmic drugs could be reduced. In one patient one ventricular tachycardia morphology from the right ventricular outflow tract was eliminated while the second ventricular tachycardia morphology was not targeted (and was suppressed by antiarrhythmic drug) for close vicinity of the arrhythmogenic focus to the left anterior descending artery. Thus the clinical benefit of the ablation procedure is enhanced to $14(87.5 \%)$ patients. Ablation completely failed in two patients.

Conclusion: Radiofrequency catheter ablation of ventricular tachycardia is highly effective and safe and results in long-term arrhythmia elimination. In patients with underlying structural heart disease it should be currently viewed as a adjunctive therapy to a complex management of the patient, while in otherwise healthy patients it can be considered a method for permanent cure.

\section{INTRODUCTION}

Sustained ventricular tachyarrhythmias represent the most life-threatening disturbance of cardiac rhythm. Many underlying structural heart diseases, generally associated with fibrous scar formation and geometrical alteration of the ventricles, predispose to the reentrant pattern of electrical activation, which is notorious arrhythmic mechanism in these conditions. Continuing ischemia, evolving ventricular function depression and limited understanding of the arrhythmogenic substrate mandates complex clinical approach to these patients. For the ventricular tachyarrhythmia itself implantation of cardioverter-defibrillator (ICD) is used as a front- 
line therapy as studies support prolonged sudden cardiovascular death survival and total survival compared to treatment with empiric or electrophysiologically guided antiarrhythmic drugs including amiodarone ${ }^{1}$. Simultaneous complex cardiological management with cardiac revascularization, beta-blocker, ACE inhibitors and hypolipidemic therapy is inherent part of the patients management. The impact of catheter ablation of ventricular tachycardia(s) (VT) on survival in patients with structural heart disease is unknown and therefore only rarely the catheter ablation alone can be considered a method for cure $2,3,4,5$. It is usually reserved for frequent symptomatic drug resistant episodes of the arrhythmia in patients with ICD already implanted ${ }^{6,7}$. It might probably safely serve as a potential therapeutic option for those patients without ICD, in whom antiarrythmic drugs failed to suppress the arrhythmia or even as a primary strategy, provided the targeted clinical VT is hemodynamically stable, the patient has no history of syncopi or cardiac arrest and no other ventricular tachyarrhythmias leading to hemodynamic compromise are inducible during the electrophysiological study. From the technical aspect, the indication criteria are mainly influenced by the hemodynamic consequences and patient's tolerance during the tachycardia mapping.

The clinical approach differs in heterogeneous group of VT in patients without obvious structural heart disease, who have generally good prognosis and in whom only rare cases of sudden death were identified in long term, usually after antiarrhythmic drug discontinuation ${ }^{8}$, $9,10,11$. The efficacy of medical antiarrhythmic therapy is relatively good with $30-35 \%$ recurrence rate of symptomatic $\mathrm{VT}^{10,11}$. The catheter ablation is mostly successful and can be used as a first-line approach or a least before the decision to provide the patient with potential danger, side effects and discomfort of long-life antiarrhythmic therapy. The efficacy of the catheter ablation in the two most prevalent entities of "idiopathic" VT reaches $90 \%$ for the right outflow tract VT (Fig. 1) when data from several series are compiled ${ }^{12,13,14,15,16}$, and is even higher, close to $100 \%$, for the VT from the region of posterior fascicle in the left ventricle (Fig. 2) ${ }^{13,}$ $16,17,18,19$.

\section{PATIENTS}

Between June 1997 and April 200023 patients (9 females) aged $49.1 \pm 15.6(18-72)$ years underwent 30 catheter ablation procedures for 29 distinct morphologies of monomorphic VT and for polymorphic VT in one case.

\section{Patients with structural heart disease}

Seven patients ( 2 females), aged $54.2 \pm 19.8$ (21-72) years, had underlying heart disease. Four of these patients had a history of remote myocardial infarction and one patient presented with polymorphic VT that occurred during the recovery phase from recent myocar- dial infarction. One of these patients also underwent earlier cardiac surgery for rheumatic valve disease with replacement of mitral valve and valvuloplasty of the aortic valve and two patients had previous coronary artery bypass graft. In one patient right ventricular arrhythmogenic dysplasia was confirmed by cardiac imaging methods and one patient with transposition of great arteries underwent Rastelli correction and subsequent reoperations with allograft replacement and excision of subvalvular aortic stenosis in the past. (details in Table 1).

Two patients were referred for catheter ablation attempt after amiodarone failed to suppress inducibility of the clinically documented VT during the electrophysiological study. Five patients were indicated to catheter ablation for frequent recurrences of VT on ineffective empirical antiarrhythmic therapy. All patients with monomorphic VT were relatively hemodynamically stable during the clinical VT episode or VT induced in the laboratory and had no history of cardiac arrest or syncope. In one patient with frequent daily episodes of polymorphic VT degenerating into ventricular fibrillation, catheter ablation was indicated as a last resort following 200 ICD shocks within the last 3 months associated with progressive reduction of left ventricular function despite repeated adjustments of medical therapy and previous surgical revascularization. In fact, this patient underwent the ablation procedure at time of severe clinical signs of left ventricular dysfunction with long-term hypotension necessitating hemodynamic support with dopamine.

\section{Patients without structural heart disease}

In sixteen patients ( 7 females) aged $44.2 \pm 12.8$ (18-66) years no obvious structural heart disease could be identified during the diagnostic work-up. The patients were often symptomatic for years and took ineffectively one or more antiarrhythmic drugs. Seven patients experienced syncopi, four patients presented with palpitation and hypotension, 3 patients complained of palpitations accompanied with fatigue and dyspnea and 2 patients had only palpitations. (details in Table 2 ).

Ten patients had VT morphology of left bundle branch block with right axis deviation suggesting right ventricular outflow tract origin (Fig. 3). Five of these patients had documented sustained and one patient incessant nonsustained symptomatic VT, four patients had frequent symptomatic episodes of nonsustained VT and one patient with less symptomatic short nonsustained VT was examined for professional reasons (pilot). In two of these patients intramural or epicardial septal origine of the VT was suspected from the surface ECG, the latter of the patients had three different VT morphologies documented during the electrophysiological study in another hospital. One patient presented with incessant long-life ventricular bigeminy and syncope. Morphology of the ectopy pointed to a different origin in the right ventricle than the outflow tract. 
Five patients had VT from the left ventricle, all with right bundle branch block morphology and left axis deviation (Fig. 4). Three of these monomorphic tachycardias were incessant nonsustained or sustained and two were paroxysmal sustained. Two of the patients experienced syncope, two had prolonged hypotension during incessant VT a one patient was relatively mildly symptomatic with VT incessant for many years.

Table 1.

\begin{tabular}{|l|l|l|l|l|l|l|}
\hline Pt/age & SHD & Other diseases & LV function & Coronary artery & Revascularization & Symptoms \\
\hline $\mathrm{m} / 71$ & MI inf & AH & Inf dyskin, EF 50 & $\begin{array}{l}\text { RCA + LAD cl, Cx 80, } \\
\text { LMB 90 }\end{array}$ & RIA, ACD, RMS & palp, ap, ht \\
\hline $\mathrm{m} / 60$ & MI inf & ASLE & Inf akin, EF 35 & ACD cl & - & palp, dys, presyn \\
\hline $\mathrm{f} / 70$ & MI as & Chron. pancr. & ApS akin, EF 15 & $\begin{array}{l}\text { LAD cl, Cx 50, RCA, } \\
80\end{array}$ & MIDCAB - RIA & ICD shocks, syn \\
\hline m/35 & ARVD & Cholecystolit. & norm & norm & - & palp, naus, vom \\
\hline m/21 & TGA & Compartment & norm & norm & - & palp, fat \\
\hline m/72 & MI inf & Stroke & Inf dyskin, EF 40 & & - & palp, fat \\
\hline f/55 & MI ap & Mi rep., Ao pl. & Ap akin, EF 45 & Cx 60 & - & palp, fat \\
\hline
\end{tabular}

Explanations: pt - patient; LV - left ventricle; SHD - structural heart disease; $\mathrm{m}$ - male; f - female; MI - myocardial infarction; inf - inferior; as - anteroseptal; ap - apical; AH - aterial hypertension; ASLE - aterosclerosis of lower extremities; Chron. pancr. - chronic pancreatitis; Cholecystolit. - cholecystolithiasis, Compartment - compartment syndrome; Mi rep. - mitral valve replacement; Ao pl. - aortic valvuloplasty, akin - akinesis; dyskin - dyskinesis; ApS - apicoseptal; EF - left ventricular ejection fraction; palp - palpitations; ap - angina pectoris; ht - hypotension; dys - dyspnea; presyn - presyncope; syn - syncope; fat - fatigue; naus - nausea, vom - vomitus, cl - closed, RCA - right coronary artery, LAD - left anterior descending artery, Cx - Circumflex artery, CMB - left marginal branch.

Table 2.

\begin{tabular}{|c|c|c|c|c|c|c|}
\hline Pt/age & Disease & Echocardiography & MR & Coronary angio & Years/Drug & Symptoms \\
\hline $\mathrm{m} / 47$ & psoriasis & TT - norm & - & - & 16/prop & palp, fat, dys \\
\hline $\mathrm{f} / 60$ & $\mathrm{DM}$ & TE - norm & norm & norm & 9/amio & palp, syn \\
\hline $\mathrm{f} / 29$ & Sine & TE - norm & norm & - & 2/amio & palp, fat \\
\hline $\mathrm{f} / 50$ & Sine & TE - norm & - & SPECT norm & $4 /$ sine & palp, syn \\
\hline $\mathrm{m} / 40$ & Sine & TT - MR I & - & - & 23/mex & palp, dys, syn \\
\hline $\mathrm{m} / 41$ & Sine & TE - RV,"RA border & norm? & - & $5 /$ sine & palp \\
\hline $\mathrm{m} / 61$ & Sine & TT - norm & & norm & $0 /$ sine & syn, subdur \\
\hline $\mathrm{m} / 46$ & Sine & TE - norm & norm & norm & $2 /$ sine & palp, syn \\
\hline $\mathrm{m} / 55$ & Sine & TT - norm & norm & norm & $0 /$ sot & palp, ht \\
\hline $\mathrm{f} / 38$ & Sine & TE - susp ARVD & norm & - & 1/metop & palp, syn \\
\hline $\mathrm{f} / 44$ & post lef AP abl & TT - norm & - & - & $4 /$ sot & palp \\
\hline $\mathrm{f} / 18$ & Sine & TT - norm & - & - & 6/prop & palp \\
\hline $\mathrm{m} / 66$ & Sine & TT - norm & - & norm & $6 /$ sot & palp, ht \\
\hline $\mathrm{f} / 52$ & Sine & TT - norm & - & norm & 1/prop & palp, ht \\
\hline $\mathrm{m} / 31$ & Sine & TE - norm & norm & norm & 1/prop & palp, ht \\
\hline $\mathrm{m} / 35$ & Sine & TE - norm & - & - & 1/prop & syn \\
\hline
\end{tabular}

Explanations: MR - magnetic resonance; coronary angio - coronary angiography; DM - diabetes mellitus; TT - transthoracic; TE - transesophageal; MR I - mitral regurgitation grade I, RA - right atrium, RV - right ventricle; border - borderline size; ARVD - arrhythmogenic right ventricular dysplasia; drug - last drug; prop - propafenon; amio - amiodarone; sot - sotalol; mex - mexiletin; metop - metoprolol; subdur - subdural hematoma; AP - accessory pathway, abl - ablation others as in table I. 
Table 3.

\begin{tabular}{|c|c|c|c|c|c|c|c|c|}
\hline Pt/age & VT morph & VT rate & Abl result & Fluoro/min & FU/mo & $\begin{array}{l}\text { Drugs/ICD } \\
\text { preabl }\end{array}$ & Drugs/ICD postabl & VT postabl \\
\hline $\mathrm{m} / 71$ & 2 & 160 & elim & 21 & 21 & amio 200 & amio 200 & no \\
\hline $\mathrm{m} / 60$ & 1 & 150 & elim & 4 & 19 & amio 200 & amio $200 /$ ICD & no \\
\hline \multirow[t]{2}{*}{$\mathrm{f} / 70$} & polymorp & & & 20 & & amio 200 / ICD & & \\
\hline & & & & 54 & 17 & & amio 200 / ICD & reduced \\
\hline \multirow[t]{2}{*}{$\mathrm{m} / 35$} & 1 & 200 & slowed & 39 & & atenolol 50 & & \\
\hline & & 165 & elim & 10 & 18 & & atenolol 50 / ICD & no \\
\hline $\mathrm{m} / 21$ & 2 & 170 & elim & 21 & 17 & sotalol 240 & sine & no \\
\hline $\mathrm{m} / 72$ & 1 & & stopped & 27 & 10 & amio 200 & amio 200 & no \\
\hline \multirow[t]{2}{*}{$f / 55$} & 2 & & $\begin{array}{l}\text { slowed, 1 VT } \\
\text { elim }\end{array}$ & 46 & & amio 300 & & \\
\hline & & & slowed & 61 & 8 & & amio 300 & 1 recur. \\
\hline
\end{tabular}

Explanations: VT - ventricular tachycardia; morph - morphology; abl - ablation; fluoro - fluoroscopy time; FU - follow-up; mo - months; preabl - preablation; postabl - postablation; polymorp - polymorphic; elim - eliminated; recur - recurrence, ICD - implantable cardioverterdefibrillator; others as in table I and II.

Table 4.

\begin{tabular}{|l|l|l|l|l|l|l|l|}
\hline Pt/age & VT documented & VT during ablation & Abl result & Fluoro/min & FU/mo & AA & VT \\
\hline $\mathrm{m} / 47$ & NSVT incess, $185 \mathrm{bpm}$ & RVOT sept, NSVT & elim NSVT+VPB & 17 & 34 & 0 & 0 \\
\hline $\mathrm{f} / 60$ & SVT, $200 \mathrm{bpm}$ & RVOT sept, NSVT & elim NSVT+VPB & 13 & & 0 & SVT \\
\hline & SVT, $200 \mathrm{bpm}$ & RVOT ant, NSVT & elim NSVT not VPB & 42 & 23 & sot 320 & NSVT \\
\hline $\mathrm{f} / 29$ & NSVT, $160 \mathrm{bpm}$ & RVOT sept, NSVT & elim NSVT, not VPB & 38 & & 0 & NSVT \\
\hline & NSVT short & RVOT ant, NSVT & elim NSVT, not VPB & 17 & 24 & prop 450 & VPB \\
\hline $\mathrm{f} / 50$ & VPB & RVOT sept, NSVT & elim NSVT, not VPB & 11 & 26 & 0 & 0 \\
\hline $\mathrm{m} / 40$ & NSVT inces, $190 \mathrm{bpm}$ & LVApS, NSVT inces & elim NSVT+VPB & 15 & 25 & 0 & 0 \\
\hline $\mathrm{m} / 41$ & VPB & RVOT as & elim NSVT+VPB & 15 & 16 & 0 & VPB \\
\hline $\mathrm{m} / 61$ & SVT inces, $170 \mathrm{bpm}$ & RVOT ant, SVT & elim SVT & 8 & 14 & 0 & 0 \\
\hline $\mathrm{m} / 46$ & SVT, $170 \mathrm{bpm}$ & LV PS, SVT & VT stopped, not elim & 65 & & & \\
\hline & & LV PS, SVT & VT slowed to 130 & 58 & 13 & prop & VT \\
\hline $\mathrm{m} / 55$ & SVT inces, $160 \mathrm{bpm}$ & LV Ap, ApS, NSVT & elim VT + VPB & 35 & 13 & sine & 0 \\
\hline $\mathrm{f} / 38$ & & RVOT & elim NSVT, not VPB & 30 & 12 & betax & 0 \\
\hline $\mathrm{f} / 44$ & & RVOT sept, delta-like & elim NSVT, not VPB & 31 & 11 & sot 160 & NSVT \\
\hline $\mathrm{f} / 18$ & NSVT inces, $125 \mathrm{bpm}$ & LV ApS, NSVT inces & elim NSVT+VPB & 11 & & sine & NSVT \\
\hline & & LV ApS, NSVT inces & elim NSVT+VPB & 7 & 9 & sine & 0 \\
\hline $\mathrm{m} / 66$ & SVT $220 \mathrm{bpm}$ & $\begin{array}{l}\text { RVOT ant, OT sept }- \\
\text { SVT, LVOT-NSVT }\end{array}$ & elim RVOT VT & 34 & 4 & amio & 0 \\
\hline $\mathrm{f} / 52$ & SVT 200 & RVOT ant, SVT inces & elim VT+VPB & 43 & 4 & sine & 0 \\
\hline $\mathrm{m} / 31$ & SVT 170 & LV ApS & elim VT & 23 & 2 & sine & 0 \\
\hline $\mathrm{m} / 35$ & VPB inces & RV baslat & elim VPB & 20 & 2 & sine & 0 \\
\hline
\end{tabular}

Explanations: AA - antiarrhythmic drugs; SVT - sustained ventricular tachycardia; NSVT - nonsustained ventricular tachycardia; VBB - ventricular premature beat; inces - incessant; RVOT - right ventricular outflow tract; LV - left ventricle; sept - septal; ant - aneterior; Ap - apical; ApS - apicoseptal; OT - outflow tract; LVOT - left ventricular outflow tract; betax - betaxolol; others as in previous tables. 


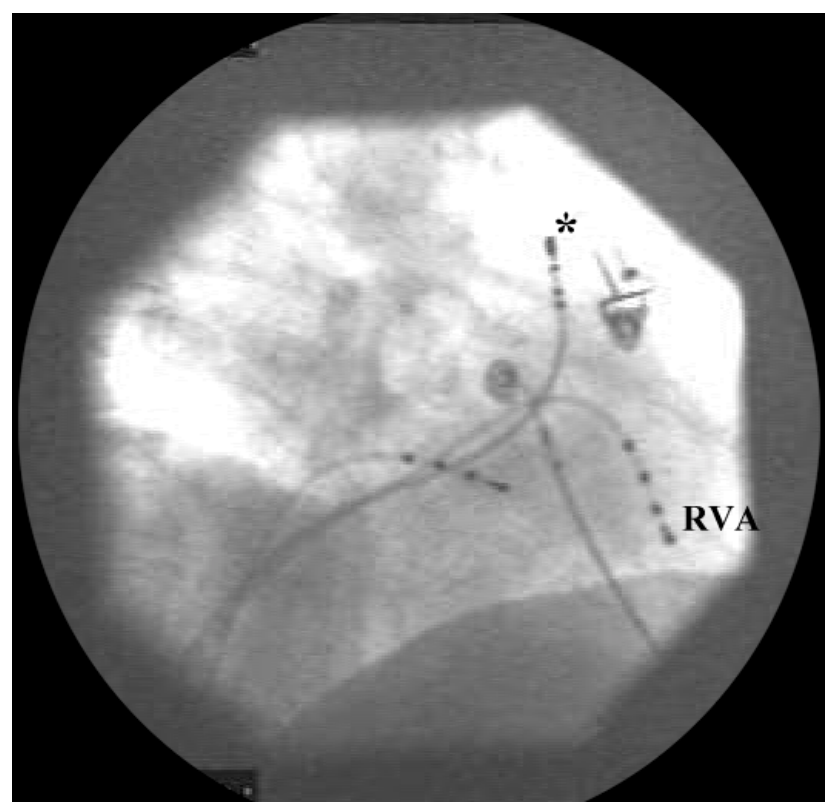

Fig. 1 Fluoroscopy image of the catheter position during mapping of the right ventricular outflow tract tachycardia in the RAO projection. Ablation catheter marked with asterix is in the right ventricular outflow tract under the pulmonic valve where the ventricular tachycardia originated. RVa denotes the catheter positioned in the right ventricular apex.

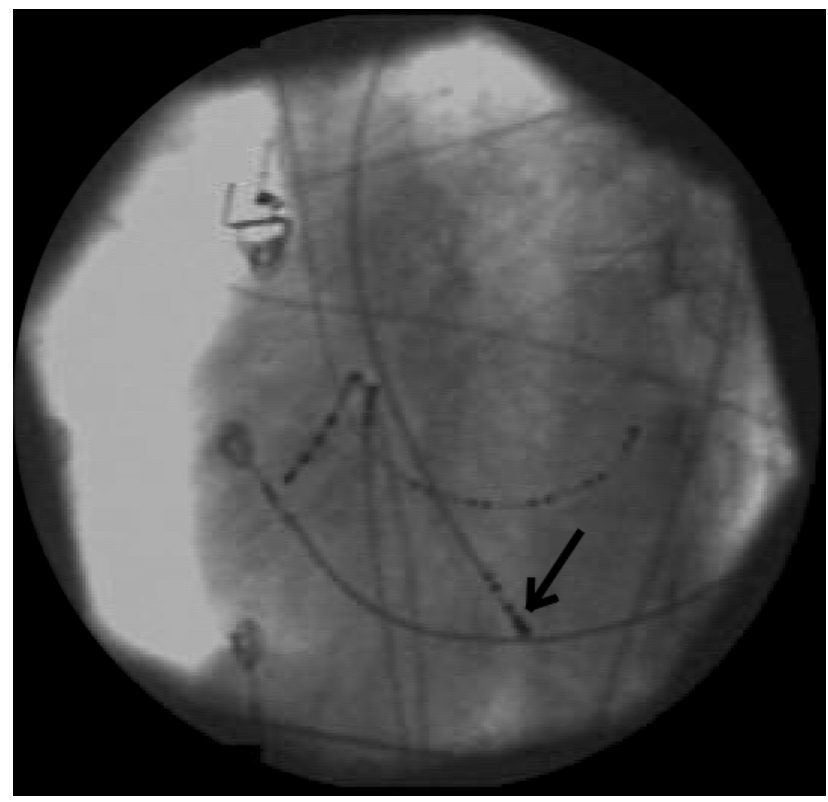

Fig. 2 Fluoroscopy image of the catheter position during mapping of the "idiopathic" left ventricular tachycardia from the inferoapicoseptal region (arrow) in the LAO projection. Catheter introduced from the superior vena cava is positioned in the coronary sinus. The third catheter is in the His bundle region.

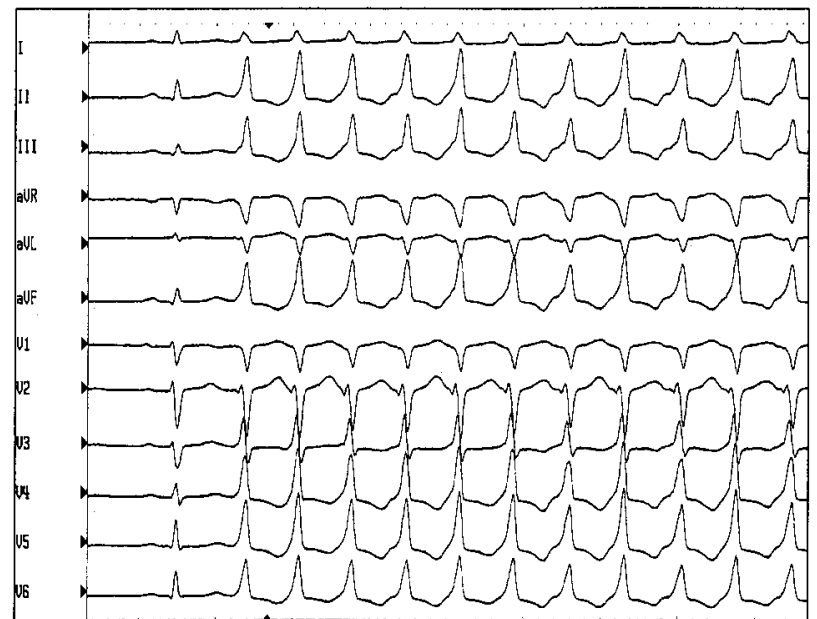

Fig. 3 Example of paroxysmal sustained monomorphic right ventricular outflow tract tachycardia in patient without structural heart disease. After the first sinus beat ventricular tachycardia starts with typical left bundle block morphology in the precordial leads (negative QRS complex in lead V1) and right axis deviation in the frontal plane (highly positive QRS complex in leads II, III, aVF, negative QRS complex in aVL).

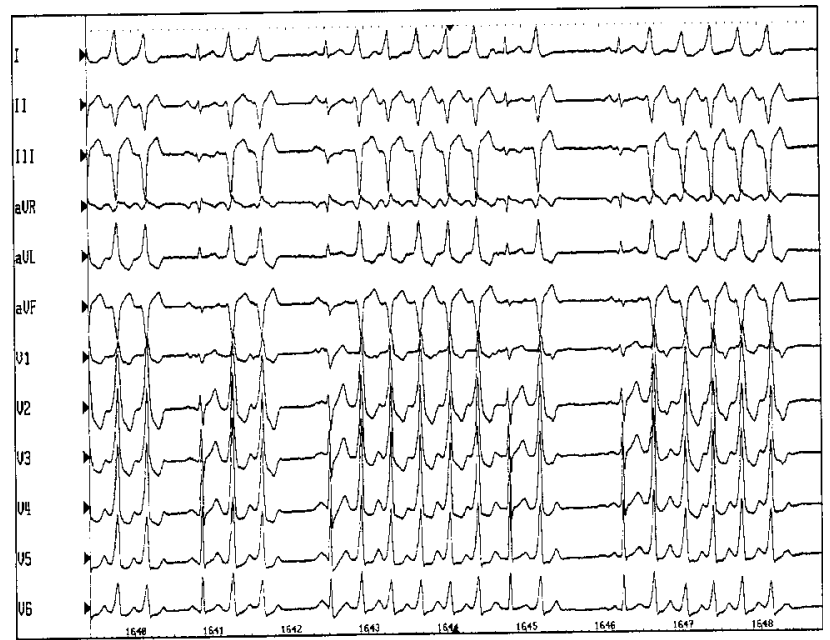

Fig. 4 Example of incessant nonsustained "repetitive" ventricular tachycardia from the inferoapicoseptal region in the left ventricle in patient without structural heart disease. Isolated sinus beats are interrupted by short bursts of ventricular tachycardia with right bundle branch morphology in the precordial leads (positive QRS complex in lead V1) and left axis deviation in the frontal plane (positive QRS complex in leads I, VL and negative QRS complex in leads II, III, $\mathrm{aVF})$. 


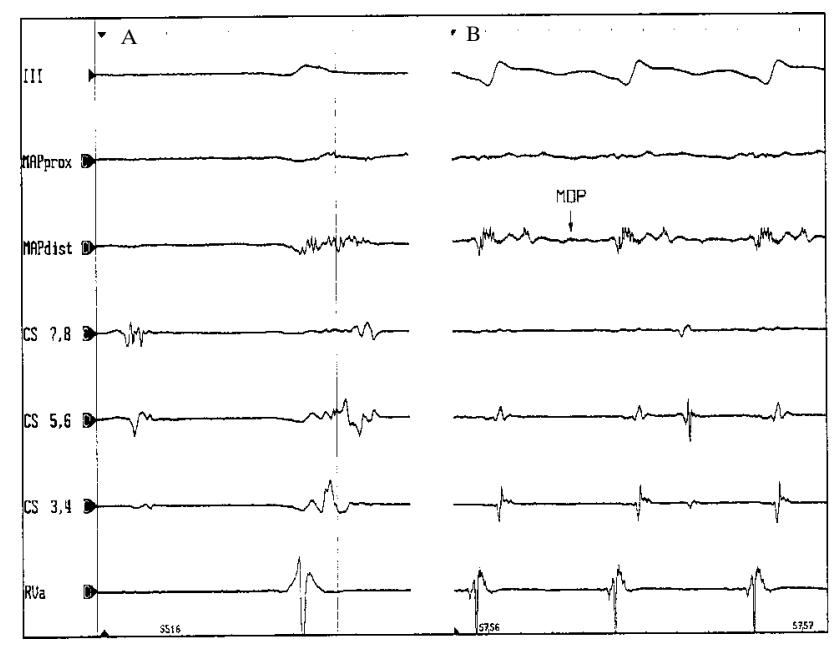

Fig. 5 Left ventricular endocardial mapping during sinus rhythm and ventricular tachycardia in a patient with remote myocardial infarction and inferior aneurysm in the left ventricle.

A: During the sinus rhythm vertical line shows the end of the QRS complex in the surface ECG leads. Notice long fractionated low voltage amplitude signal mapped in the basoinferior wall of the left ventricle between the aneurysm and the mitral annulus (MAP dist). This abnormal potential extends beyond the end of the QRS complex (late potential) and can be seen also in adjacent recordings from the coronary sinus on the mitral annulus (CS 7,8, CS 5,6).

B: After induction of ventricular tachycardia with the ablation catheter at the site of late potential (see A) notice almost continuous fractionated activity with a potential in the early diastolic phase and small fractionated potential in the middiastolic phase (MDP in MAPdist). These abnormal potentials during sinus rhythm and the ventricular tachycardia represent slow conduction in the functionally altered myocardium at the edge of the postmyocardial infarction scar responsible for the ventricular tachycardia existence. Recording speed $200 \mathrm{~mm} / \mathrm{s}$.

Abbreviations: MAPprox, MAPdist - recordings from the proximal and distal bipole of the mapping/ablation catheter; CS 7,8-3,4 - bipolar recordings from the catheter in the coronary sinus (from the septum towards the lateral wall); $\mathrm{RVa}$ - bipolar recording from the right ventricular apex.

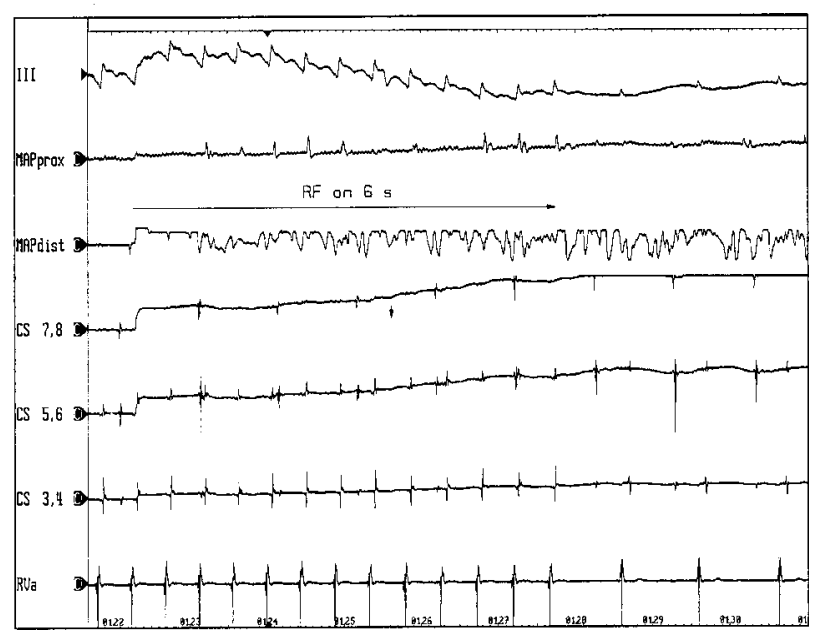

Fig. 6 Termination of the ventricular tachycardia with radiofrequency energy delivery. During radiofrequency ablation at the site from fig. 4 the tachycardia stopped and was rendered noninducible. Recording speed $25 \mathrm{~mm} / \mathrm{s}$. Abbreviations as in figure 4 .

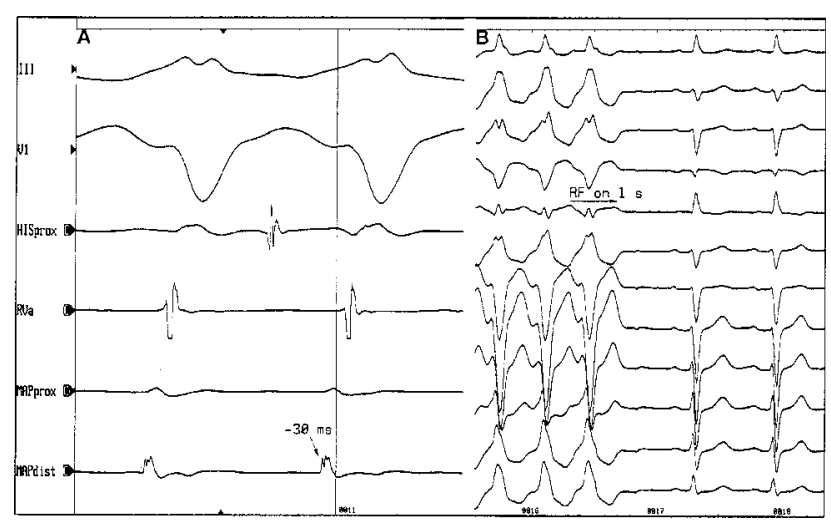

Fig. 7 Endocardial mapping of right ventricular outflow tract ventricular tachycardia and radiofrequency catheter ablation.

A: Earliest activation (site of origine of the ventricular tachycardia) during the tachycardia is in the right ventricular outflow tract under the pulmonic valve (MAP dist) and precedes the QRS complex on the surface ECG by $30 \mathrm{~ms}$. This local potential is relatively sharp without diastolic activity or presystolic fractionated activity. Activation in the right ventricular apex (RVa) is late. Proximal bipole of the catheter in the His bundle region (HISprox) shows atrial potential which is dissociated from the ventricular activity. (Recording speed $200 \mathrm{~mm} / \mathrm{s}$ ) B: Surface ECG recording during radiofrequency energy delivery at the site of earliest endocardial activation ( see A). The tachycardia terminated within one second and became noninducible. (Recording speed $50 \mathrm{~mm} / \mathrm{s}$ ).

Abbreviations: MAPdist, MAPprox - recordings from distal and proximal bipole of the mapping/ablation catheter; RVa - recording from the catheter in the right ventricular apex; Hisprox - recording from the proximal bipole of the catheter in the His bundle region.

\section{METHODS}

During the electrophysiological study usually one $6 \mathrm{~F}$ quadripolar or multipolar catheter was introduced into the right ventricle for mapping and pacing, one $6 \mathrm{~F}$ quadripolar catheter was positioned in the His bundle region and one 5 or $6 \mathrm{~F}$ decapolar catheter into the coronary sinus. Occasionally, one thin $2 \mathrm{~F}$ catheter was introduced into a venous branch of the coronary sinus for epicardial left ventricular mapping. A 7F steerable quadripolar catheter with $4 \mathrm{~mm}$ tip electrode was introduced into the left or right ventricle for mapping and radiofrequency energy delivery. In one case a $7 \mathrm{~F}$ catheter with saline irrigated and cooled $4 \mathrm{~mm}$ tip electrode was employed to enable deeper lesion.

Bipolar endocardial or epicardial electrograms were digitally recorded and stored on a Cardiolab System (Prucka Engineering) and radiofrequency current generators HAT 200S (Osypka) or Stockert (Cordis-Webster) were used for radiofrequency ablation.

Inducibility and reproducibility of the targeted VT were extensively evaluated even if they were known from previous electrophysiological study. This was important for further evaluation of immediate ablation result. In case of a reentry tachycardia in structural heart disease, abnormal late potentials following the terminal part of QRS complex (Fig. 5A) on the surface ECG were looked for to roughly identify regions of slow 
conduction at the border of myocardial infarction scar. Then the VT was induced and abnormal fractionated low amplitude potentials in the diastolic phase (Fig. 5B) were indicative of the slow conduction area responsible for the VT maintenance. Ideally, entrainment with concealed fusion was used to confirm, that this slow conduction area is a part of the reentry circuit and not a dead-end bystander. The endpoint of the ablation procedure included termination of the VT with radiofrequency current delivery (Fig. 6) and its further noninducibility. In one patient with polymorphic VT a different encircling approach was employed with step by step radiofrequency energy applications around the myocardial infarction scar with the endpoint to eliminate or substantially decrease all abnormal late potentials during the sinus rhythm.

In patients with normal heart, in whom the mechanism of VT is widely nonreentrant, infusion of isoproterenol was often needed to induce and sustain the arrhythmia. Still, in some patients only nonsustained VT episodes could be observed. In other patients either sustained paroxysms of VT could be induced by pacing or incessant tachycardia was present and enabled straightforward arrhythmia mapping. If the VT was sustained enough, mapping of the earliest activation (Fig. 7) was started and, if necessary, combined with pacemapping during the sinus rhythm. In nonsustained VT mainly pacemapping was relied on to guide the radiofrequency energy application.

\section{RESULTS}

\section{Patients with structural heart disease}

One patient after inferior myocardial infarction underwent ablation of the isthmus between the postinfarction scar and mitral annulus with elimination of two VT morphologies and remains free of VT recurrence for 21 months on previously ineffective amiodarone. In second patient with remote inferior myocardial infarction and several recent VT episodes on amiodarone medication the arrhythmia was eliminated with a single radiofrequency delivery. Although ventricular fibrillation was later inducible during the electrophysiological study and ICD was implanted, this patient did not have any recurrence of ventricular arrhythmia for 19 months. In another patient with remote inferior myocardial infarction and spontaneous VT episodes, which were also inducible despite amiodarone therapy, the VT was only terminated but not eliminated during RF energy delivery. Although the procedure had to be prematurely stopped with incomplete result for patient's intolerance to stay in rest in supine position, no VT recurrence occurred during the 10 month period. In one patient after myocardial infarction and surgery for valve disease with frequent recurrences of monomorphic VT on amiodarone, two morphologies of VT were targeted around the apical aneurysm. One of the VT with electrophysiological signs of intramyocardial or epicardial ori- gin was not eliminated. This arrhythmia was terminated several times but, as a result of the procedure, was only slowed during the second ablation with a cooled tip ablation catheter, which enables larger radiofrequency lesion. This patient experienced one recurrence of slowed well-tolerated VT within 8 month after the last ablation procedure. The patient with polymorphic VT after recent myocardial infarction dramatically improved with reduction of ICD discharges from 200 per 3 months to 70 per 17 months. She also improved hemodynamically with reduction of rehospitalizations to 5 within the follow-up period. The patient with arrhythmogenic right ventricular dysplasia and frequent VT episodes had the clinical VT eliminated by linear lesion between the abnormal tissue in the outflow tract and the pulmonary valve. Although he also received ICD for different fast VT morphologies inducible during the electrophysiological study, he did not experienced any VT recurrence on unchanged betablocker therapy for 17 months. The patient after surgical correction of transposition of great arteries, who presented with frequent episodes of VT on sotalol is completely free of VT recurrences without antiarrhythmic medication for 16 months (details in Table 3).

\section{Patients without structural heart disease}

In 4 patients with inducible sustained or incessant nonsustained monomorphic right ventricular outflow tract tachycardia, which was easily mapped during the electrophysiological study, all of these tachycardias were eliminated with catheter ablation and did not recur. Three of these patients are free of antiarrhythmic medication. In the fourth patient two more different monomorphic tachycardia morphologies from the ventricular outflow tract were inducible, one sustained and one nonsustained. The sustained VT originated epicardially close to the left anterior descending artery. This VT was not targeted by radiofrequency current for the risk of the coronary artery closure. The latter VT was only nonsustained arising probably from left ventricular outflow tract according to the surface ECG criteria and was not mapped. Both the arrhythmias were suppressed by amiodarone and rendered noninducible.

In six patients only nonsustained ventricular tachycardia could be induced during the electrophysiological study. The ectopic rhythm was eliminated in two patients and significantly suppressed in one patient, who are free of symptoms without antiarrhythmic medication. In two patients accurate mapping during the electrophysiological study was difficult due to multiple morphology of ventricular ectopy. In these patients targeting larger area in the right ventricular outflow tract led to significant reduction of the ectopic rhythm and enabled to discontinue amiodarone in both the patients. In one patient with surface ECG criteria for deep intramural or epicardial septal origin, ablation attempt from the right endocardial aspect failed.

Three incessant VTs arising from the left posterior fascicle region were eliminated by radiofrequency abla- 
tion. Fascicular potential preceding the earliest endocardial ventricular activation was found at the ablation site in one case. Reablation for VT recurrence was required several months later in one of these patients and was successful. In one patient sustained monomorphic VT with similar morphology and anatomical origin in the inferoapicoseptal region, local electrical signal exhibited marked late potentials during sinus rhythm and long diastolic fractionated activation during the VT. This electrocardiographic pattern along with possibility to entrain the VT, and inducibility of second VT morphology originating from the same arrhythmic substrate during the study, suggested reentry mechanism. Structural changes in the ventricles, however, were not confirmed by extensive examination including coronary angiography and magnetic resonance. All the patients remain free of palpitations and antiarrhythmic drugs. In one patient with VT originating in the left ventricle posteroseptally under the mitral valve, the arrhythmia could be only terminated by radiofrequency current delivery, but was not eliminated after two ablation procedures. Later this patient underwent unsuccessful catheter ablation with the same findings in a different hospital. Mapping data and response to radiofrequency ablation suggested deep intramyocardial arrhythmic substrate not accessible to the conventional ablation equipment (details in Table 4).

\section{DISCUSSION}

Results of radiofrequency catheter ablation in this series of patients with sustained monomorphic ventricular tachycardia suggest relatively high immediate success rate and encouraging long-term clinical outcome.

In group of patients with underlying structural heart disease the catheter ablation led to elimination and noninducibility of all targeted clinical sustained monomorphic VTs in $4(67 \%)$ out of 6 patients. In one patient, one VT morphology was eliminated and noninducible during the first procedure and the second VT morphology originating deeper in the myocardium became significantly slower and less well inducible after second ablation attempt. In the last patient the tachycardia was successfully mapped and stopped with radiofrequency current, but the patient s intolerance (previous stroke and residual hemiparesis) to stay longer in supine position prevented complete elimination of the larger arrhythmogenic substrate.

The acute ablation success rate is similar to the results of previous larger series of patients, who underwent catheter ablation of postinfarction sustained monomorphic $\mathrm{VT}^{4,5,7}$. In these series acute success of ablation procedure, which was around $70 \%$, included both the patients, in whom none targeted VT morphology was inducible at the end of the study, as well as patients with modified, but still inducible VT. There is scarce data on catheter ablation of monomorphic VT in the setting of arrhythmogenic right ventricular dysplasia, however smaller studies suggest acute success rate not exceeding $50 \%{ }^{20,21}$. There has been no case of VT ablation after surgical correction of transposition of great arteries described in the literature.

During the long-term follow-up five of the patients, all with successful ablation and one with incomplete ablation, had no recurrence of any ventricular tachyarrhythmia on previously ineffective or even discontinued antiarrhythmic therapy and thus the clinical benefit of the ablation is enhanced to $5(83.3 \%)$ patients. The patient with deep intramural or epicardial origin of the VT experienced one episode of slower and well-tolerated VT of unchanged morphology in the sixth month of the eight month follow-up period. The clinical results in the two patients with incomplete immediate success suggest at least alteration of the arrhythmogenic substrate rendering the tachycardia less susceptible to occur. Two of the patients had nonclinical polymorphic VT and/or ventricular fibrillation inducible during the electrophysiological study after catheter ablation of the clinical monomorphic VT. ICD was implanted in both the patients. None of them had a ventricular tachyarrhythmia detected and treated by the ICD discharges.

Long-term follow-up in previous studies of postinfarction VT patients indicates around 60\% arrhythmia free survival ${ }^{4,5,7}$ in patients with successful ablation, while three quarters of patients, in whom ablation failed, experienced VT recurrence within four weeks of ablation $^{5}$. The clinical outcome was even better (91\% patients free of arrhythmia) in patients, who had not previous failed antiarrhythmic drug therapy. However, these patients formed highly selected group with welltolerated VT and benign clinical history ${ }^{5}$. On the other hand difficult issue is faced in patients, who are receiving amiodarone at the time of completely successful ablation. As amiodarone may suppress other VT morphologies than that aimed with catheter ablation, it seems wise to continue the amiodarone therapy unless ICD is implanted ${ }^{5}$. Patients with modified or complex arrhythmogenic substrate, who have other rapid nonclinical VT morphologies inducible after successful elimination of clinical VT should probably receive ICD, which is supported by observation of almost $50 \% \mathrm{VT}$ recurrences during the follow-up period in this subset of patients ${ }^{4}$.

One patient with recent myocardial infarction deteriorated hemodynamically as a result of continuing ischemia not suitable for repeated revascularization and of incessant polymorphic VT with multiple discharges from ICD a day for the last three months. As a last resort, after many adjustments of pharmacologic therapy, ablation of the arrhythmogenic substrate with successive radiofrequency deliveries around the infarction scar during sinus rhythm was attempted and led to early hospital discharge and significant ICD shocks reduction as well as reduction of rehospitalizations.

Previous studies showed yield of catheter ablation for ICD therapy reduction in patients with frequent monomorphic $\mathrm{VT}^{6,7}$. In patients with hemodynamically 
unstable VT that cannot be mapped during the VT several ablation strategies are under developement (unpublished data) usually employing electroanatomical mapping with Carto system (Cordis-Biosense) which creates three-dimensional color map of the electrical activation sequence in the heart chambers ${ }^{22}$. These strategies use voltage mapping to identify scar, normal myocardium and abnormal tissue on the scar edge and try to transect this abnormal scar with radiofrequency current to interrupt possible reentry circuits. Clinical benefit of this ablation approach is not known at present.

In long-term all patients benefit from the catheter ablation intervention. This favorable clinical outcome is enhanced by relatively reasonable fluoroscopy times and uncomplicated course of the ablation procedure in this subset of patients with cardiovascular diseases.

In patients with VT, who have no obvious structural heart disease, the immediate and long-term ablation results are excellent in $7(77,7 \%)$ out of 9 patients, in whom the tachycardia could be sustained or was present in nonsustained but incessant form, which helped mapping during the ablation procedure. In 4 patients with incessant or inducible sustained VT originating in the inferoapicoseptal region of the left ventricle, and in 3 patients with inducible sustained or incessant VT from the right ventricular outflow tract, the VT was eliminated and the patients are free of palpitations and antiarrhythmic drugs. In one patient with sustained right outflow tract VT which was successfully eliminated with radiofrequency current, a different VT morphology originating epicardially close to the proximal part of left anterior descending artery was inducible but not ablated for safety reasons. This VT resistant to many antiarrhythmic drugs including sotalol, was suppressed and rendered noninducible by amiodarone. In one patient, tachycardia originating deep intramyocardially or epicardially in the posteroseptal region of the left ventricle could only be stopped but not eliminated and ablation with conventional $4 \mathrm{~mm}$ tip catheter failed in our and later in a different hospital.

In six patients the right ventricular outflow tract VT was seen only in the form of relatively sporadic nonsustained VT during the electrophysiological study. Two of these patients had documented sustained VT in the past accompanied by syncope. Three patients have the ectopy eliminated or significantly suppressed and do not take antiarrhythmic drugs in long term. Two patients underwent second ablation procedure for a different morphology of the ectopy from the right ventricular outflow tract and in none of them the ectopy was eliminated completely. Reduction of the ectopy enabled replacement of amiodarone for small dose of sotalol resp. propafenon, which is clinical benefit in these patients. Delta-like morphology and early transition of the ectopic QRS complex in the precordial leads support epicardial origin of nonsustained repetitive VT in one patient in whom ablation from the right endocardial approach failed. This patient continues to take sotalol with partial effect. One patient with permanent ventricular bigeminy was examined for the history of syncope associated with palpitations. Nonsustained VT was induced with isoproterenol and was provoked by radiofrequency energy delivery. In this patient ablation of a focus in the basolateral right ventricular wall, guided by mapping of ventricular premature beats, led to complete elimination of the ectopy and symptoms.

Our results are similar to general experience with short- and long-term outcome ranging between $70 \%$ to $100 \% 12,13,14,16,17,18$. Recent experience shows rather less successful results which may reflect indications in wider spectrum of idiopathic VT foci originating also in the left ventricular outflow tract as well as deep intramyocardial or epicardial foci in the outflow tract. Moreover, occassional VTs were described to arise from the sinuses of Valsalva ${ }^{22,23}$. These arrhythmogenic foci may not be accessible to conventional ablation therapy or are not approached by radiofrequency current for the risk of coronary artery damage. Recently ablative cure of symptomatic ventricular ectopic beats was attempted by some authors ${ }^{24,25,26}$. It is also our experience that mapping of isolated ventricular extrasystoles may be difficult particularly if the morphology of the ectopy changes during the ablation procedure and consequently the clinical outcome may not be ideal.

\section{CONCLUSION}

Catheter ablation of ventricular tachycardia is effective in majority of patients and can be completed safely also in patients with advanced structural heart disease. In the setting of previous myocardial infarction and other cardiomyopathies associated with anatomicofunctional changes of the myocardium and modulating conditions such as e.g. ischemia, one has to consider complexity and instability of the ensuing arrhythmogenic substrate. Therefore catheter ablation in these patients offers only adjunctive therapy which, however, can significantly influence quality of life and potentially the survival. On the other hand, ventricular tachycardia arising from smaller arrhythmogenic foci in otherwise healthy myocardium can be permanently cured by catheter ablation without long-term need for further medical care. Failure to eliminate the arrhythmia is usually a consequence of technical limitations of contemporary equipment to reach and destroy arrhythmogenic substrate which is large or epicardially located. New catheter design and three-dimensional nonfluoroscopy mapping are expected to enhance the accuracy and efficacy of the method together with marked reduction of radiation dose. 


\section{REFERENCES}

1. The Antiarrhyhmics Versus Implantable Defibrillators (AVID) Investigators: A comparison of antiarrhythmic drug therapy with implantable defibrillators in patients resuscitated from near fatal ventricular tachyarrhythmias. N. Engl. J. Med., 1997, 337, 1576-1583.

2. Morady, F., Harvey, M., Kalbfleish, S.J., et al. (1993) Radiofrequency catheter ablation of ventricular tachycardia in patients with coronary artery disease. Circulation, 87, 363-372.

3. Gonska, D. B., Cao, K., Schaumann, A., et al. (1994) Catheter ablation of ventricular tachycardia in 136 patients with coronary artery disease: results and long-term follow-up. J. Am. Coll. Cardiol., 24, 1506-1514.

4. Rothman, S. A., Hsia, H. H., Cossú, S. F., et al. (1197) Radiofrequency catheter ablation of postinfarction ventricular tachycardia: Long-term success and the significance of inducible nonclinical arrhythmias. Circulation, 96, 3499-3508.

5. Stevenson, W. G., Friedman, P. L., Kocovic, D., et al. (1198) Radiofrequency catheter ablation of ventricular tachycardia after myocardial infarction. Circulation, 98, 308-314.

6. Willems, S., Borggrefe, M., Shenasa, M., et al. (1993) Radiofrequency catheter ablation of ventricular tachycardia following implantation of an automatic cardioverter defibrillator. PACE, $16,1684-1692$.

7. Strickberger, A. S., Man, K. C., Daoud, E. G., et al. (1997) A prospective evaluation of catheter ablation of ventricular tachycardia as adjuvant therapy in patients with coronary artery disease and an implantable cardioverter-defibrillator. Circulation, 96, 1525-1531.

8. Buxton, A. E., Waxman, H. L., Marchlinski, F. E., et al. (1983) Right ventricular tachycardia: Clinical and electrophysiologic characteristics. Circulation, 68, 917-927.

9. Deal, B.J., Miller, S.M., Scagliotti, D., et al. (1986) Ventricular tachycardia in a young population without overt heart disease. Circulation, 73, 1111-1118.

10. Lemery, R., Brugada, P., Della-Bella, P., et al. (1989) Nonischemic ventricular tachycardia: Clinical course and long-term follow-up in patients without clinically overt heart disease. Circulation, 79, 990-998.

11. Goy, J. J., Tauxe, F., Fromer, M., et al. (1990) Ten-year follow-up of 20 patients with idiopathic ventricular tachycardia. PACE, 13, 1142-1147.

12. Klein, L. S., Shih, H. T., Hackett, K., et al. (1992) Radiofrequency catheter ablation of ventricular tachycardia in patients without structural heart disease. Circulation, 85, 1666-1674.

13. Goggins, D. L., Lee, R .L., Sweeney, J., et al. (1994) Radiofrequency catheter ablation as a cure for idiopathic tachycardia of both left and right ventricular origin. J. Am. Coll. Cardiol., 23 $1333-1341$.
14. O’Connor, B. K., Case, C. L., Sokolovski, M. C., et al. (1996) Radiofrequency catheter ablation of right ventricular outflow tract tachycardia in children and adolescents. J. Am. Coll. Cardiol., 27, 896-874.

15. Movsowitz, C., Schwartzman, D., Callans, D. J., et al. (1996) Idiopathic right ventricular outflow tract tachycardia: Narrowing the anatomic location for successful ablation. Am. Heart J., 131, 930-936.

16. Rodriguez, L. M., Smeets, J. L. R. M., Timmermans, C., et al. (1997) Predictors for successful ablation of right- and left-sided idiopathic ventricular tachycardia. Am. J. Cardiol., 79, 309-314.

17. Nakagawa, H., Beckman, K. J., McClelland, J. H., et al. (1993) Radiofrequency catheter ablation of idiopathic left ventricular tachycardia guided by a Purkinje potential. Circulation, 88 , 2607-2617.

18. Wen, M. S., Yeh, S. J., Wang, C. C., et al. (1994) Radiofrequency therapy in idiopathic left ventricular tachycardia with no obvious structural heart disease. Circulation, 89, 1690-1696.

19. Wen, M. S., Yeh, S. J., Wang, C. C., et al. (1994) Successful radiofrequency ablation of idiopathic left ventricular tachycardia at a site away from the tachycardia exit. J. Am. Coll. Cardiol., 30, 1024-1031.

20. Stark, S. I., Arthur, A., Lesh, M. D. (1996) Radiofrequency catheter ablation of ventricular tachycardia in right ventricular cardiomyopathy: Use of concealed entrainment to identify the slow conduction isthmus bounded by an aneurysm and the tricuspid annulus. J. Cardiovasc. Electrophysiol., 7, 967-971.

21. Ellison, K. E., Friedman, P. L., Ganz, L. I., et al. (1998) Entrainment mapping and radiofrequency catheter ablation of ventricular tachycardia in right ventricular dysplasia. J. Am. Coll. Cardiol., 32, 724-728.

22. Shimoike, E., Ohba, Y., Yanagi, A., et al. (1998) Radiofrequency catheter ablation of left ventricular outflow tract tachycardia: Two case reports. J. Cardiovasc. Electrophysiol., 9, 196-202.

23. Shimoike, E., Yasushi, O., Ueda, M., et al. (1999) Radiofrequency catheter ablation of left ventricular outflow tract tachycardia from the coronary cusp: A new approach to the tachycardia focus. J. Cardiovasc. Electrophysiol., 10, 1005-1009.

24. Zhu, D. W. I., Maloney, J. D., Simmons, T. W., et al. (1995) Radiofrequency catheter ablation for management of symptomatic ventricular ectopic activity. J. Am. Coll. Cardiol., 26, 843-849.

25. Lauck, G., Burkhardt, D., Manz, M. (1999) Radiofrequency catheter ablation of symptomatic ventricular ectopic beats originating in the right ventricular outflow tract. PACE, 22, 5-16.

26. Seidl, K. H., Schumacher, B., Hauer, B., et al. (1999) Radiofrequency catheter ablation of frequent monomorphic ventricular ectopic activity. J. Cardiovasc. Electrophysiology, 10, 924-934. 